\title{
PHASE NOISE SQUEEZING BASED PARAMETRIC BIFURCATION TRACKING OF MIP-COATED MICROBEAM MEMS SENSOR FOR TNT EXPLOSIVE GAS SENSING
}

\author{
L. L. Li ${ }^{I^{*}}$, E. L. Holthoff ${ }^{2}$, L. A. Shaw ${ }^{1}$, C.B. Burgner ${ }^{l}$ and K.L. Turner ${ }^{l}$ \\ ${ }^{1}$ University of California, Santa Barbara, California, USA \\ ${ }^{2}$ Army Research Laboratory, Sensors and Devices Directorate, Adelphi, MD, USA
}

\begin{abstract}
This paper reports real time explosive gas sensing (DNT) in atmospheric pressure utilizing the noise squeezing effect that occurs prior to a bifurcation event. A noise-squeezing controller based on the statistics of phase noise is implemented using high speed LabVIEW ${ }^{\mathrm{TM}}$ field programmable gated array (FPGA). A high frequency TNT-molecularly imprinted fixed-fixed microbeam sensor utilizes this nontraditional sensing strategy and performs DNT sensing at various concentrations. Experiments are conducted using both noise-based and sweep-based bifurcation tracking for a direct comparison. Results demonstrate noise-based bifurcation tracking is not only capable of performing reliable frequency tracking, but also shows the method is superior to the bifurcation sweep-based tracking. Over three orders of magnitude improvement in acquisition rate is achieved, and, as a result, confidence and precision on bifurcation frequency estimation is significantly improved over the bifurcation sweep tracking method, enabling DNT sensing at concentrations much below sub-ppb (parts-per billion) level.
\end{abstract}

\section{INTRODUCTION}

High sensitivity and selectivity and low cost are key considerations for sensors designed for trace explosive detection [1]. Low mass, high frequency and low cost micro/nano sensors utilizing mass loading of microcantilevers have drawn increasing attention in the area of mass sensing in recent years [2,3]. Molecular imprinted polymers (MIPs) have become an attractive thin-film coating for many MEMS sensors and a deeper understanding of binding sites in MIPs has been achieved $[4,5]$. In this work, $15 \mathrm{~nm}$ films of sol-gel-derived xerogels molecularly imprinted for tri-nitro toluene (TNT) have demonstrated selectivity and stability in combination with a fixed-fixed beam MEMS sensor [6-7]. The sensor was characterized by parametric bifurcation sweep-based tracking [6]. Traditionally, mass sensing using MEMS has been achieved based on the natural frequency shift due to an increase of resonator mass. However, noise has set the limit of detection for linear sensing [8]. The ability to track the minimum shift of the natural frequency is determined by both the intrinsic and extrinsic noise of the system. Dynamics of parametrically excited oscillators and their applications have been studied extensively [9-11] as well as successful attempts to improve the effective quality factor of microcantilever arrays operating in the linear regime utilizing parametric amplification have been made $[12,13,15]$. However, bifurcation mass sensing has demonstrated superior sensitivity in the presence of measurement noise when compared to linear sensing in air [14]. As shown in figure 1, the critical location of the bifurcation mass tracking is recorded by repeatedly sweeping the frequency towards the critical point until large amplitude occurs. A system reset is required after each sweep cycle due to hysteresis. This sensing method offers higher sensitivity and noise resistivity over the harmonic resonant tracking [14], however, the long settling time and its high dependency on sweep rate to noise ratio [16-17] make it less desirable. In this work, we investigate a different sensing approach based on noise squeezing effect in parametric systems $[15,18-20]$ that occurs prior to the critical point. A LabVIEW ${ }^{\mathrm{TM}}$ FPGA controller is implemented to keep the device operating close to the edge of instability and inhibiting large amplitude grows (Fig. 1). As a result, close to three order of magnitude of improvement in acquisition rate is achieved.

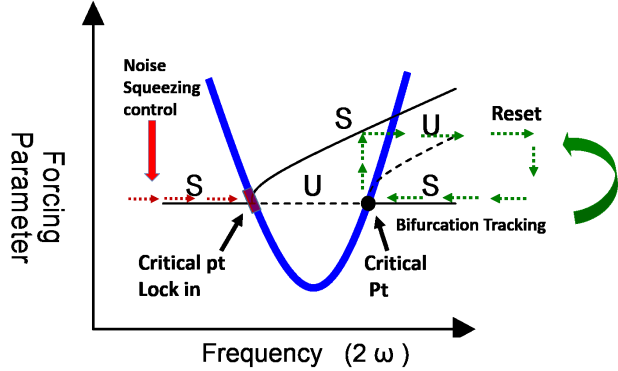

Figure 1: Schematic of both noise squeezing-based tracking (left) and bifurcation sweep-based tracking methods (right). The bifurcation sweep based tracking method tracks the bifurcation locations by repeatedly performing frequency sweeps towards critical point until large amplitude results. Then the device is relaxed to a zero stable state before the next sweep starts. However, in the noise squeezing control tracking method, the device approaches the critical point until the noise squeezes below some threshold, then feedback control keeps the device close to the edge of instability while maintaining small a response amplitude.

\section{THEORY}

In this work, parametric resonance is achieved by applying periodic axial forcing to a fixed-fixed microbeam through an external shear piezo, and the resulting dynamic is governed by the Matehieu-Hill equation with noise term, and the analysis can be found in [21]. As shown in figure 2, far away from the critical point where zero solution is stable, the phase appears to be random ranging from $-\pi$ to $\pi$ due to thermomechanical noise. As it gets closer to the critical point, phase noise squeezes onto the slow manifold that corresponds to the eigenvector direction associated with eigenvalue with the smallest magnitude. If it keeps going, the phase noise squeezes more and zero solution becomes unstable, large amplitude results. The large phase variance where phase starts to squeeze makes it a perfect location for a feedback controller operation to stay close to the edge of bifurcation (Figure $1)$.

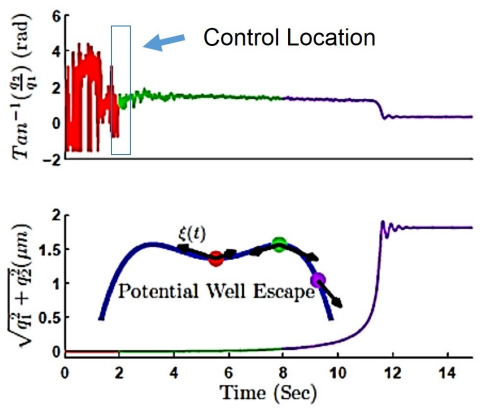

Solid-State Sensors, Actuators and Microsystems Workshop Hilton Head Island, South Carolina, June 8-12, 2014 
Figure 2: Transient Response of a parametrically excited gyro before and after the critical bifurcation point. A coordinate transformation of $\left(q_{1}, q_{2}\right)$ into a frame rotating at half the drive frequency is used. The system undergoes a supercritical/subcritical pitchfork bifurcation. Far away from the critical point, the phase (in red) alternates randomly with a large variance. As the device approaches the critical point, indicated by the transition from red to green, the dynamics collapse onto a one dimensional slow manifold, where the phase variance drops dramatically, making it an ideal location for control. As a consequence, the phase noise correlates and the amplitude starts to grow. Escape to a large amplitude (in purple) occurs as the parameters cross the instability boundary.

\section{DEVICE AND EXPERIMENTAL SETUP}

The device under testing is comprised of a fixed-fixed microbeam with a natural frequency of $49.33 \mathrm{kHz}$, as shown figure 3. The sensor was spin-coated with xerogel-based molecularly imprinted polymers (MIPs) that is highly selective to TNT. However, 2-4 di-nitro toluene (DNT) is used for mass sensing experiment, the results can be inferred to the sensing of TNT as they are structurally similar.

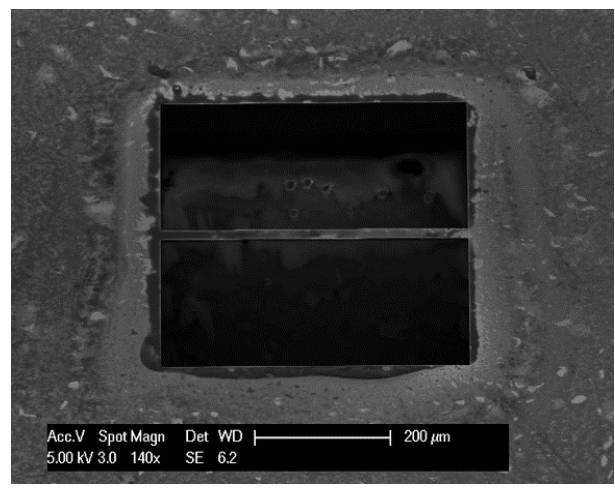

Figure 3: SEM image of a $450 \mu m \times 20 \mu m \times 2 \mu m$ sensor coated with MIPs. The device used in this paper is $600 \mu \mathrm{m} \times 20 \mu \mathrm{m} \times 2 \mu \mathrm{m}$.

Shown in Figure 4, experiment is conducted at atmospheric pressure and room temperature. The sensor was mounted on a shear piezo, driven by the function generator at nearly twice the resonant frequency (to drive parametric resonance) at a fixed voltage of 32 Volts. DNT vapor was generated in a permeation oven by heating 2-4 DNT permeation tube at constant temperature mixed with $\mathrm{N}_{2}$. The flow rates were controlled by mass flow controllers (MFC). The concentration of the DNT/ $\mathrm{N}_{2}$ mixture in parts per billion (ppb) can be calculated based on the flow rate and oven temperature for a specific permeation tube. The sensor response is detected by an optical laser vibrometer and the signal is analyzed in the phase lock amplifier, which outputs the amplitude and phase in reference to the reference signal at half the drive frequency. The FPGA controller samples the phase data and is dedicated to the calculation of phase variance. Based on the statistics of the phase variance, a feedback control is implemented to adjust the corresponding frequency change to keep the device close to the edge of instability. Any change of frequency due to mass loading is followed by the noise squeezing controller immediately.

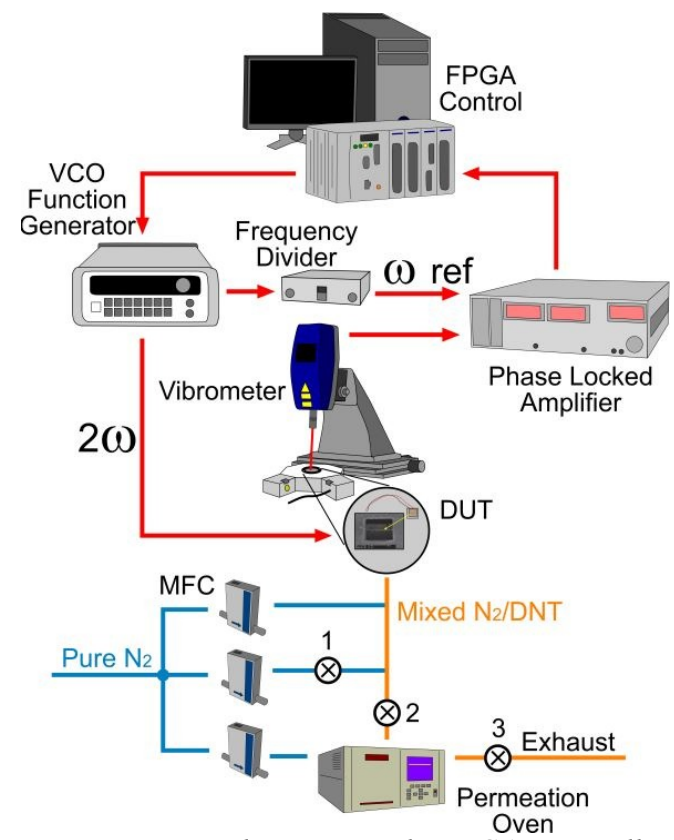

Figure 4: Experimental setup and FPGA controller design schematics. The device is mounted onto a shear piezo and is driven at $\sim$ twice its natural frequency. The sensor velocity response coupled by a laser vibrometer through an optical microscope. The vibrometer signal and the reference square wave at half the drive frequency are fed into a phase lock amplifier (PLA). The FPGA samples the outputs of the PLA and provides feedback control to hold response close to the edge of bifurcation. The 2-4 DNT/N $\mathrm{N}_{2}$ gas test experiment is conducted in a closed chamber at atmospheric pressure and room temperature. DNT/N $N_{2}$ is generated by heating the 2-4 DNT permeation tube in a permeation oven at constant temperature. DNT vapor is carried by $\mathrm{N}_{2}$ and the mixture is fed into the test chamber at constant flow rate controlled by mass flow controllers. When the device is not under active DNT test, only $\mathrm{N}_{2}$ is used.

\section{RESULTS}

DNT gas sensing experiment was performed with the noisesqueezing controller at a low concentration of $0.93 \mathrm{ppb} \mathrm{DNT} / \mathrm{N}_{2}$ mixture. In this experiment, nine repeated tests of 15 minutes of pure $\mathrm{N}_{2}$ followed by 10 minutes of $\mathrm{DNT} / \mathrm{N}_{2}$ were conducted, as shown in Figure 5.

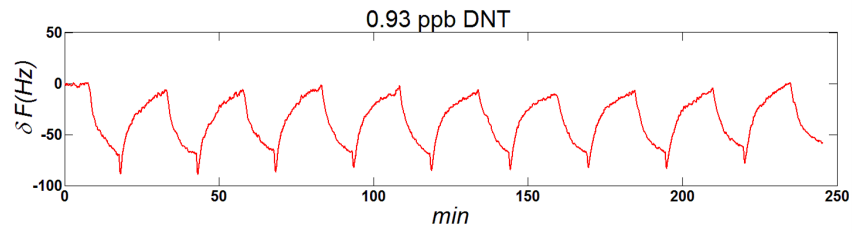

Figure 5: Figure shows a constant concentration of $0.93 \mathrm{ppb}$ $D N T / N_{2}$ gas experiment. Pure $N_{2}$ was first introduced for 15 minutes and was followed 10 minutes of $\mathrm{DNT} / \mathrm{N}_{2}$. The parametric drive frequency was lowered by $67 \pm 3 \mathrm{~Hz}$ due to the polymer absorption of DNT. The same process was repeated 9 times. The linear frequency drift of $0.05 \mathrm{~Hz} / \mathrm{min}$ was accounted for in the post processing.

Higher concentration DNT gas experiments were also conducted. These experiments were carried out by the noise squeezing bifurcation sensing method and the bifurcation sweep tracking method described in [7] for comparison. Reversibility 
was not able to obtained due to the stiction of DNT at high concentrations, hence, instead of introducing $\mathrm{N}_{2}$ purging cycle after each DNT testing, higher concentration DNT experiment were conducted after the previous concentration reached its saturation level. Calibration curve of frequency shift as a function of the concentration in parts per billion (ppb) shown in Figure 8 is used to find the sensitivity of the MIPs. Frequency stability is quantified by Allan Variance [22] of data highlighted in red in Figure 6 and Figure 7. The sensitivity of frequency shift to concentration is characterized by the slope of the calibration curve in Figure 8. A summary of the comparison of the two tracking method is shown in table 1 .
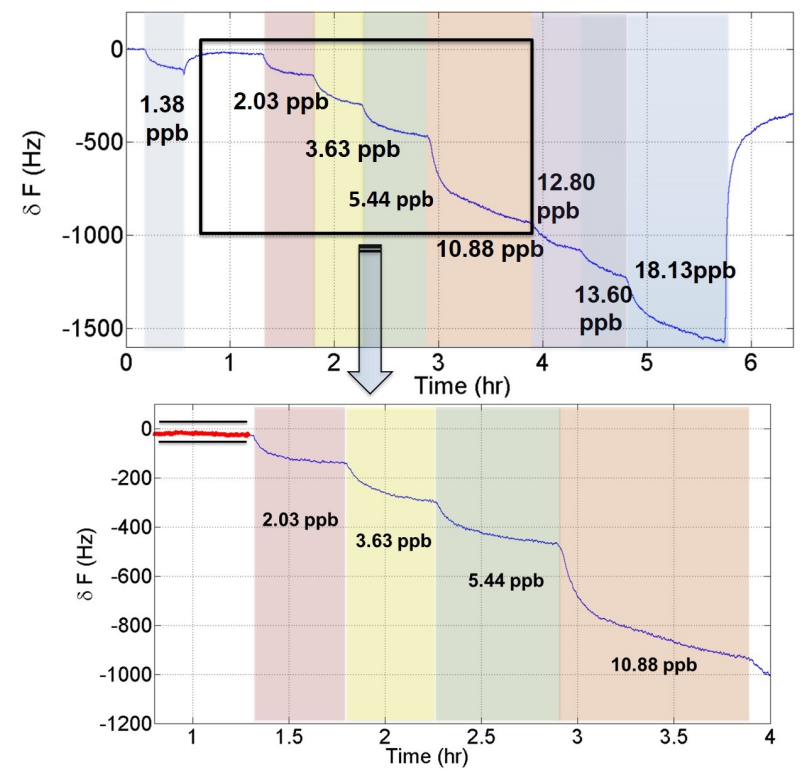

Figure 6: DNT gas sensing using a noise squeezing controller. (a) shows the gas experiment with lowest concentration of $1.38 \mathrm{ppb}$ and highest concentration of $18.13 \mathrm{ppb}$. (b) is a zoom in figure of the portion inside the square window in (a). The highlighted portion corresponds to pure $\mathrm{N}_{2}$ purging after the $1.38 \mathrm{ppb} \mathrm{DNT/N_{2 }}$ gas test. However, absorbed DNT did not completely come off, and the frequency did not fully recover initial starting frequency. Hence, this explains why the highlighted data in red does not start at zero. (b) is used to make direct comparison with the data collected from the bifurcation sweep method (Figure 7) at the same concentration.

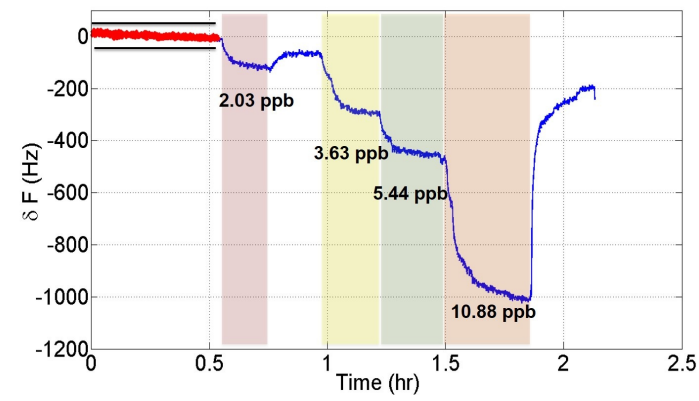

Figure 7: DNT gas sensing using the bifurcation sweep method. Pure Ns was introduced after the first and last DNT gas test (2.03 ppb and $10.88 \mathrm{ppb}$ ). Absorption phenomenon persisted, and the following higher concentration $D N T / N_{2}$ experiments were conducted after the steady state of lower concentration was reached.

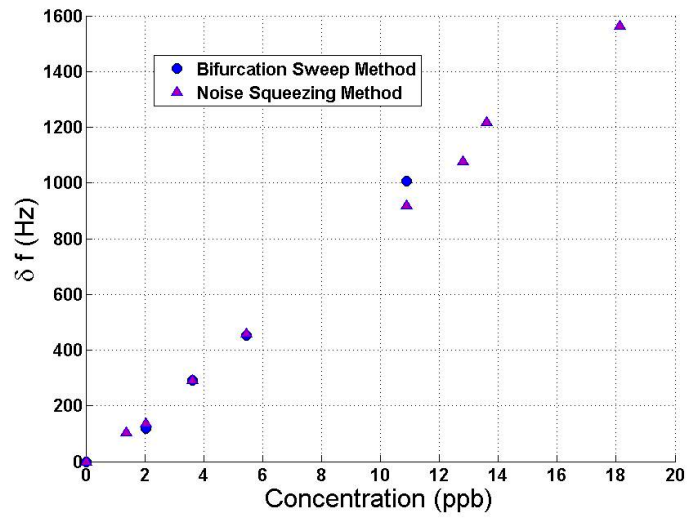

Figure 8: Calibration curve for both the noise squeezing tracking and bifurcation sweep tracking method. The slope of linear curve fit for each corresponds to its sensitivity.

Table 1: This table summarizes the results of both the noise squeezing method and the bifurcation method. Minimum frequency corresponds to the Allan variance for each method and sensitivity corresponds to the slopes of the calibration curve. LOD is the ratio between minimum frequency and the sensitivity of natural frequency.

\begin{tabular}{|l|l|l|}
\hline & $\begin{array}{l}\text { Noise } \\
\text { Squeezing } \\
\text { Method }\end{array}$ & $\begin{array}{l}\text { Bifurcation } \\
\text { Sweep } \\
\text { Method }\end{array}$ \\
\hline Minimum Frequency (Hz) & 0.045 & 5.43 \\
\hline Sensitivity (Hz/ppb) & $87.62 \pm 3$ & $94.7 \pm 13.5$ \\
\hline LOD (ppb) & 0.0005 & 0.06 \\
\hline
\end{tabular}

\section{DISCUSSION}

Experimental results demonstrate that noise-squeezing control bifurcation tracking is capable of tracking frequency change due to mass loading. The lowest concentration tested is $0.93 \mathrm{ppb}$, and good reversibility is demonstrated at low concentration (Figure 5). The noise squeezing controller estimates the critical location at less than $10 \mathrm{~ms}$, while the average time for a bifurcation sweeps take up to $15-20 \mathrm{~s}$. Hence, acquisition rate is increased by close to three orders of magnitude. The noise squeezing controlled tracking offers better frequency stability over the bifurcation sweep tracking method, leading to two orders of magnitude smaller in lowest order of detection (LOD). The DNT response time is found to be around $90 \mathrm{sec}$, which is much longer than the reported time in [7]. This can be attribute to the degradation of the polymer, since it was over two years old when the experiments were conducted for this paper. This issue is also reflected in the sensor's inability to recover to its original state at high concentration shown in Figure 6 and Figure 7. However, the DNT response sensitivity is directly related to the sensitivity of the polymer. Hence, confidence of the noise squeezing controlled bifurcation tracking ability can still be assured.

\section{CONCLUSION}

Experiments successfully demonstrate that the noise squeezing based sensing is a superior sensing strategy over the sweep-based bifurcation tracking method for real time DNT explosive sensing at atmospheric pressure. Three orders of magnitude improvement in 
acquisition rate leads to faster, more confident and more precise estimation over the compared sensing method [23]. Even though the sensor response to the TNT is slow due to the degrading of the MIPs, it does not question the sensitivity of the noise-squeezing controller, since the TNT/DNT sensitivity of the sensor is highly depended on the sensitivity of the coating. This issue is expected to be resolved with better coating material and is currently underway.

\section{ACKNOWLEDGEMENT}

This work was supported by the Institute for Collaborative Biotechnologies through grant W911NF-09-0001 from the U.S. Army Research Office. The content of the information does not necessary reflect the position or the policy of the Government and no official endorsement should be inferred. Travel support has been generously provided by the Transducer Research Foundation.

\section{REFERENCES}

[1] Senesac, L., \& Thundat, T. G. (2008). Nanosensors for trace explosive detection. Materials Today, 11(3), 28-36. doi:10.1016/S1369- 7021(08)70017-8

[2] Lang, H. P., Berger, R., Battiston, F., Ramseyer, J.-P., Meyer, E., Andreoli, C., ... Gimzewski, J. K. (1998). A chemical sensor based on a micromechanical cantilever array for the identification of gases and vapors. Applied Physics A: Materials Science \& Processing, 66(7), S61-S64. doi:10.1007/s00339005110

[3] Battiston, F. ., Ramseyer, J.-P., Lang, H. ., Baller, M. ., Gerber, C., Gimzewski, J. ., ... Güntherodt, H.-J. (2001). A chemical sensor based on a microfabricated cantilever array with simultaneous resonance-frequency and bending readout. Sensors and Actuators B: Chemical, 77(1-2), 122-131. doi:10.1016/S0925-4005(01)00683-9

[4] Ayela, C., Vandevelde, F., Lagrange, D., Haupt, K., \& Nicu, L. (2007). Combining resonant piezoelectric micromembranes with molecularly imprinted polymers. Angewandte Chemie (International ed. in English), 46(48), 9271-4. doi:10.1002/anie.200703881

[5] García-Calzón, J. a., \& Díaz-García, M. E. (2007). Characterization of binding sites in molecularly imprinted polymers. Sensors and Actuators B: Chemical, 123(2), 11801194. doi:10.1016/j.snb.2006.10.068

[6] Holthoff, E. L., Stratis-Cullum, D. N., \& Hankus, M. E. (2011). A nanosensor for TNT detection based on molecularly imprinted polymers and surface enhanced Raman scattering. Sensors (Basel, Switzerland), 11(3), 2700-14. doi:10.3390/s110302700

[7] Turner, K. L., Burgner, C. B., Yie, Z., \& Holtoff, E. (2012). Using nonlinearity to enhance micro/nanosensor performance. In 2012 IEEE Sensors (pp. 1-4). IEEE. doi:10.1109/ICSENS.2012.6411564

[8] Ekinci K L, Yang Y T and Roukes M L 2004 J. Appl. Phys 95 2682-9

[9] Rhoads, J. F., Shaw, S. W., \& Turner, K. L. (2006). The nonlinear response of resonant microbeam systems with purely-parametric electrostatic actuation. Journal of Micromechanics and Microengineering, 16(5), 890-899. doi:10.1088/0960-1317/16/5/003

[10] Rhoads, J. F., Shaw, S. W., Turner, K. L., Moehlis, J., DeMartini, B. E., \& Zhang, W. (2006). Generalized parametric resonance in electrostatically actuated microelectromechanical oscillators. Journal of Sound and Vibration, 296(4-5), 797-829. doi:10.1016/j.jsv.2006.03.009
[11] Rhoads, J. F., Shaw, S. W., \& Turner, K. L. (2010). Nonlinear Dynamics and Its Applications in Micro- and Nanoresonators. Journal of Dynamic Sy20-222stems, Measurement, and Control, 132(3), 034001. doi:10.1115/1.4001333

[12] Yie, Z., Miller, N. J., Shaw, S. W., \& Turner, K. L. (2012). Parametric amplification in a resonant sensing array. Journal of Micromechanics and Microengineering, 22(3), 035004. doi:10.1088/0960-1317/22/3/035004

[13] Cleland, A. N. (2005). Thermomechanical noise limits on parametric sensing with nanomechanical resonators. New Journal of Physics, 7(1), 235-235. doi:10.1088/13672630/7/1/235

[14] Yie, Z., Zielke, M. a, Burgner, C. B., \& Turner, K. L. (2011). Comparison of parametric and linear mass detection in the presence of detection noise. Journal of Micromechanics and Microengineering, 21(2), 025027. doi:10.1088/09601317/21/2/025027

[15] D. Rugar \& P. Grutter. Mechanical Parametric Amplification and Thermomechanical Noise Squeezing. Physics Review Letters. Vol. 67, Number 6. August 1991.

[16] Evstigneev, M. (2008). Statistics of forced thermally activated escape events out of a metastable state: most probable escape force and escape-force moments. Physical review. E, Statistical, nonlinear, and soft matter physics, 78(1 Pt 1), 011118. Retrieved from http://www.ncbi.nlm.nih.gov/pubmed/18763930

[17] Mandel, P., \& Erneux, T. (1987). The slow passage through a steady bifurcation: Delay and memory effects. Journal of Statistical Physics, 48(5-6), 1059-1070. doi:10.1007/BF01009533

[18] G. Prakah,et al. (2012) Parametric Noise Squeezing and Parametric Resonance of Microcantilevers in Air and Liquid Environments. Review of Scientific Instruments.

[19] Nicholas Miller, Chris Burgner, Mark Dykman, Steven Shaw, Kimberly Turner, Fast estimation of bifurcation conditions using noisy response data, 2010/3/25, SPIE Smart Structures and Materials+ Nondestructive Evaluation and Health Monitoring, Pages76470O-76470O-12

[20] Christopher Burgner, William Snyders, \& Kimberly Turner, Control of MEMS on the Edge of Instability, IEEE Transducers, Beijing, China June 2011

[21] Ph.D. dissertation of Nicholas J. Miller. "NOISE IN NONLINEAR MICRO-RESONATORS",

[22] D.W. Allan. Statistics of atomic frequency standards. Proc. IEEE, 54:221- 231, 1966.

[23] Li, L. L.,Holthoff, E. L, Shaw, A. L., Burgner, B. C \& Turner, K. L. "Noise Squeezing Controlled Parametric Bifurcation Tracking of MIP-Coated Microbeam MEMS sensor for TNT Explosive Gas Sensing”, JMEMS, in press.

\section{CONTACT}

*L.L.Li, lily@engr.ucsb.edu 